\title{
Preliminary clinical experience of low-level laser therapy for the treatment of canine osteoarthritis-associated pain: A retrospective investigation on 17 dogs
}

\author{
Loris Barale ${ }^{1}$, Paolo Monticelli ${ }^{2}$, Massimo Raviola ${ }^{3}$ and Chiara Adami ${ }^{2 *}$ \\ ${ }^{1}$ Freelance Veterinarian, Carmagnola, Turin, Italy \\ ${ }^{2}$ Department of Clinical Sciences and Services, Royal Veterinary College, University of London, London, UK \\ ${ }^{3}$ Clinica Veterinaria Europa, Turin, Italy
}

\begin{abstract}
Background: Osteoarthritis (OA) is common in dogs and causes chronic pain that affects the quality of life and may not respond to analgesics.

Objective: The objective of this study was to determine whether low-level laser therapy (LLLT) would improve the quality of life and help reducing systemic analgesics, in dogs with OA.

Methods: Seventeen client-owned dogs diagnosed with OA and associated pain were included. The diagnosis of OA was confirmed by orthopedic and radiographic examination. Pain was evaluated in each dog with the canine brief pain inventory (CBPI), compiled by the dog owners, as well as with a visual analog scale (VAS) and the colorado state canine chronic pain scale, used by the clinician. The LLLT was performed weekly in each study dog, for a total period of 6 weeks. The CBPI was then repeated at 2, 4, 6, and 8 weeks after the first laser session, whereas the VAS was reassessed at weeks 2 and 6 . The dogs were observed for the occurrence of laser-related side effects.

Results: Both CBPI and VAS were significantly reduced after the first laser session ( $9.2 \pm 3.8$ and $5.2 \pm 1.1$, respectively) compared to pretreatment values ( $11.8 \pm 3.6$ and $7.6 \pm 0.9$, respectively; and $p=0.018$ and $p<0.001$, respectively) and continued to decrease over time until the end of the therapy. Based on these results and improved function, as assessed by the orthopedic surgeon, the pharmacological analgesic therapy was reduced by the clinician at week 2 in 13 of 17 dogs. Laser-related side effects were not observed.

Conclusion: This retrospective report provides a basis for future investigations, needed to clarify whether laser therapy may be beneficial to treat canine OA-associated pain. The preliminary findings are promising and suggest that LLLT may help reducing the analgesic administration and improving client satisfaction and the quality of life of dogs with OA.

Keywords: Canine osteoarthritis, Chronic pain, Low-intensity laser therapy, Orthopedic pain.

\section{Introduction}

Osteoarthritis (OA) is a progressive, degenerative disease that, in the US, affects as many as $20 \%$ of dogs aged 1 year or more (Cimino Brown, 2017). The condition causes chronic pain and decreased joint function, which on the long term severely affects the quality of life. The common treatments for OAassociated pain include long-term administration of nonsteroidal anti-inflammatory drugs (NSAIDs) and nutraceuticals, potentially with the addition of gabapentinoid and opioid analgesics to address unresponsive pain (Pettitt and German, 2015). NSAIDs are associated with gastrointestinal, hepatic, and renal side effects and, often, to inadequate pain relief. The chronic use of opioids in client-owned pets, on the other hand, carries the risk for human drug abuse and, therefore, raises a number of ethical concerns.

Within the past decade, there has been an increasing interest in nonpharmacological therapy of both human and canine OAs. Among these therapies,

electroanalgesia techniques have been raising a great interest among human doctors and veterinarians. In human medicine, the most promising electroanalgesic techniques to treat $\mathrm{OA}$ are those that imply the use of laser. Within the past decade, both low-level laser therapy (LLLT) and high-level laser therapy have been used to treat human OA-associated pain with no adverse effects (Huang et al., 2015; Youssef et al., 2016; White et al., 2017). High-level laser therapy was first introduced only in 2011, and a recent systematic review that included the first six studies conducted in people found that it was effective in reducing pain and providing functional improvements in humans with knee OA (Wyszyńska and Bal-Bocheńska, 2018). Regarding the veterinary literature, one trial performed in 12 dogs investigated the effects of LLLT on bone healing and acute surgical pain after tibial plateau levelling osteotomy (TPLO), with disappointing results (Kennedy et al., 2018). To the best of the authors' knowledge, only one study carried out in 20 dogs with elbow OA reported the successful
\end{abstract}


use of laser to treat canine OA-associated chronic pain (Looney et al., 2018).

The aim of this retrospective study was to investigate the clinical usefulness of LLLT, in terms of both improvement of quality of life as perceived by the animal owners and reduction of pharmacological analgesic therapy as decided by the primary clinician, in a population of client-owned dogs diagnosed with $\mathrm{OA}$ and presented with pain.

\section{Materials and Methods}

Before commencing the laser therapy, all dogs underwent an orthopedic and radiographic examination to confirm the diagnosis of $\mathrm{OA}$ and pretreatment baseline pain assessment with the visual analog scale (VAS; range: 0-10 cm; McCormack et al., 1988). For a more comprehensive evaluation of pain and functional impairment, the dog owners were asked to compile the canine brief pain inventory (CBPI; range: 0-100; Brown et al., 2007), before the beginning of laser therapy and then $2,4,6$, and 8 weeks after that. Each time, the dog owners evaluated their own dogs independently, and the score sheet was handed to the veterinarian immediately after completion to prevent the owner from recording the results and comparing them to previous or subsequent assessments. The VAS was repeated at weeks 2 and 6 to evaluate the response to pain therapy.

For the laser therapy, a device designed for veterinary use was used (LaserVet 1000; GlobusVet, Italy). Before the treatment, the dogs were left undisturbed for 10 minutes in the examination room, to allow acclimatization and minimize stress and discomfort. The dog owners paid regular charges for the treatment and did not receive any compensation for their dog's participation in the study.

The areas of the body treated with laser were the affected joints and the associated skeletal muscle complexes, namely, the semitendinosus and semimembranosus for the stifle, the iliopsoas for the hip, and the triceps and the brachialis for the elbow. The laser probe was applied directly on the skin of the area to be treated; the joints were always treated first, before the muscles. Each area was treated weekly, for a total of 6 weeks. By using the predetermined programs, the duration of laser exposure ranged from 50 seconds to 4 minutes, depending on coat pigmentation (light or dark), and body weight (from 1 $\mathrm{kg}$ to $>25 \mathrm{~kg}$ ). Other variables were set by the software

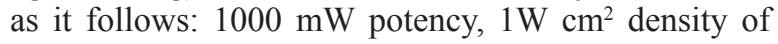
potency, 808-nm laser beam wavelength, frequencies of 500-1000 (joints) and 3,000-5,000 Hz (muscles), energy of 5 (joints) and 4.2 (muscles) $\mathrm{J} \mathrm{cm}^{2}$, and spot laser diameter varying from 3.5 to $11.5 \mathrm{~mm}$, depending on the surface area to be treated. Both continuous and pulsate emittance were used with an alternate pattern, with cycles of the same duration.

The dogs were observed after each treatment for the occurrence of side effects, namely, itch, redness, swelling, changes in skin/coat pigmentation, and any kind of discomfort perceived by either the dog owners or the clinician. After 2 weeks from the beginning of laser treatment, on the occasion of the first posttreatment pain scores, and then again at Week 8 , the pharmacological therapy was reassessed, based on the results of pain assessment, owner interview, and orthopedic examination of each dog, and adjusted, if needed, at clinician's discretion.

The Kolmogorov-Smirnov test was used to assess the data distribution. Following, the pain scores were analyzed using one-way analysis of variance, with the time point set as a grouping factor, followed by all pairwise multiple comparisons with the Holm-Sidak method. Commercially available software was used (SigmaPlot 10 and SigmaStat 3.5, SYSTAT Software Inc, CA, USA). The $p$ values $<0.05$ were considered to be statistically significant.

\section{Ethical approval}

This study was conducted under the approval of the Social Science Ethical Review Board of the Royal Veterinary College (license number: URN SR 20190238). A verbal consent for data publication was obtained by all dog owners.

\section{Results and Discussion}

The data are represented as means and SD. Seventeen dogs, 11 females (of which, 10 were neutered) and 6 males (of which, one was castrated), aged $134 \pm 34$ months and weighing $21 \pm 11 \mathrm{~kg}$, were included in this report. The represented dog breeds were mixed breed $(n$ $=6)$, Beagle $(n=2)$, German Shepherd $(n=3)$, Border Collie $(n=1)$, Pug $(n=1)$, Shetland $(n=1)$, Cane Corso $(n=1)$, Labrador Retriever $(n=1)$, and Pinscher $(n=1)$. At the time of pretreatment examination, all dogs were on pharmacological analgesic treatment since at least 2 weeks, which included meloxicam, gabapentin, and amantadine in one dog, meloxicam and gabapentin in 5 out of 17 cases, meloxicam alone in 10 out of 17 cases, and gabapentin alone in one dog. The treated joints were the hips $(n=16)$, stifle $(n=$ $7)$, elbow $(n=1)$, and lumbosacral junction $(n=1)$; 7 dogs presented with more than one affected joint. One dog, a 16-year-old mixed breed male castrated dog weighing $9 \mathrm{~kg}$, affected by chronic renal disease which suddenly deteriorated, died before Week 4 for causes unrelated to OA.

Both CBPI and VAS decreased after the first laser session compared to pretreatment baseline values and continued to decrease over time until the end of the therapy. For the CBPI, pretreatment baselines $(11.8 \pm 3.6)$ were significantly higher than the values recorded at weeks $2(9.2 \pm 3.8 ; p=0.018), 4(7.6 \pm 3.3$; $p=0.001), 6(6.8 \pm 3.5 ; p<0.001)$, and $8(4.4 \pm 4.0$; $p<0.001$ ) after treatment (Fig. 1). Similarly, the baseline VAS scores $(7.7 \pm 0.8)$ were higher than those recorded at Weeks $2(5.2 \pm 1.1 ; p<0.001)$ and $6(3.4 \pm 1.4$; $p<0.001)$ after the beginning of laser therapy. 
Canine Brief Pain Inventory (CBPI) score over time (0 - 8 weeks)

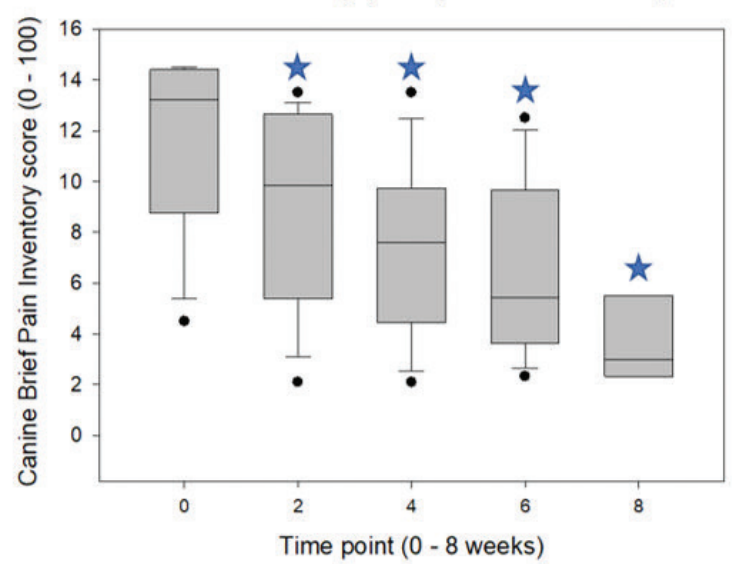

Visual Analogue Scale (VAS) score over time (0-6 weeks)

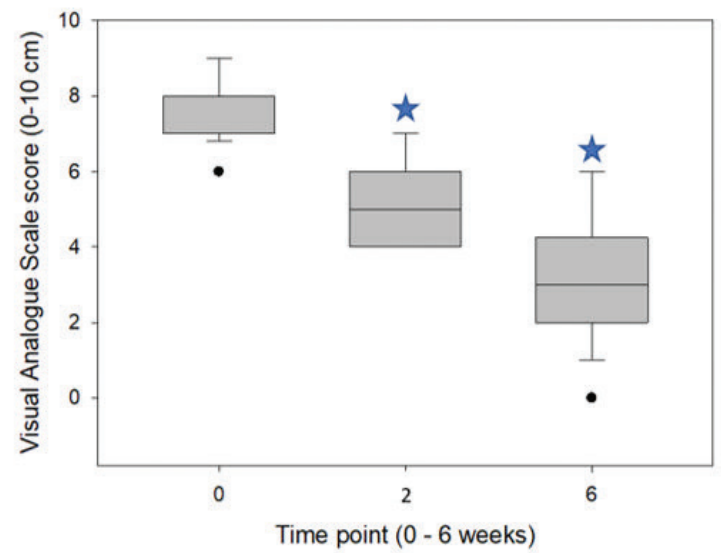

Fig. 1. The box plots represent the scores for CBPI, as evaluated by the dog owners, and VAS, as assessed by the clinician who performed the treatment, in 17 dogs with OA-associated pain undergoing laser therapy. The interquartile range boxes represent the data higher (upper quartile) and lower (lower quartile) than the medians, accounting for $50 \%$ of the total data. The whiskers are indicative of the ranges for the bottom $25 \%$ and the top $25 \%$ of the data values. The outliers are represented by the dots, and the stars indicate statistically significant differences $(p<0.05)$ between the baseline pretreatment values $(0)$ and the values recorded at the subsequent time points $(2,4,6$, and 8 on the $\mathrm{X}$-axis are weeks after the first laser therapy session, respectively; data at time point 8 are from $6 / 17$ dogs only).

After 2 weeks from the first laser session, based on the outcome (pain scores, repeated orthopedic examination, and client satisfaction), the primary clinician changed the pharmacological analgesic therapy in 15 of 17 dogs. This change consisted of a reduction in 13 of these 15 dogs, an addition in one dog, and a replacement in another one. Systemic analgesics administration was suspended in 6 out of 17 dogs and decreased in 7 out of 17 dogs; of these, four dogs previously receiving meloxicam and gabapentin had the NSAID withdrawn, whereas, in another dog, the daily dose of meloxicam was halved. In one dog previously receiving only meloxicam, the clinician added gabapentin. Finally, in another dog previously on meloxicam, the clinician replaced the NSAID with gabapentin alone. In two dogs, the administration of analgesics remained unchanged.

Only six out of 17 dogs were brought to the practice at Week 8 for a follow-up. Of these, three dogs that at Week 6 were still receiving meloxicam and gabapentin alone, respectively, and had their therapy withheld; one dog in which meloxicam dose had been halved at Week 6 had the NSAID withheld, and the remaining two dogs remained on gabapentin as at Week 6. The owners of the dogs that did not return to practice were phone interviewed and were satisfied with the clinical improvement of their pets.

No side effects of laser therapy were observed at any time in any of the study dogs.

The most relevant finding of this study was that laser therapy was effective in improving the dogs' quality of life as perceived by their owners, and it also helped decreasing the administration of systemic analgesics.
Interestingly, these positive effects could be seen immediately after the first laser therapy session and were enhanced by the repetition of the treatment over the 8-week study period.

Overall, the clients appreciated that their dogs seemed to enjoy life more and showed increased general activity and, in most cases, that the systemic administration of analgesics could be reduced. In one patient, a $30-\mathrm{kg}$ mixed breed dog with unilateral hip OA, the clinician added gabapentin to meloxicam 2 weeks after the first laser session. Based on both CBPI and VAS scores, this dog had neither improved nor worsened compared to his pretreatment condition; nevertheless, the therapy was re-evaluated and adjusted on request of the dog owner, who perceived the need for further improvement.

Despite there is convincing evidence that LLLT has a limited efficacy in improving human OA-associated pain (Huang et al., 2015), this seemed not to be the case for the dogs of this report. One reason for this may be that although the laser output used in the study dogs still falls, by definition, within the classification of low-level, the device was set to deliver its output at the highest ranges of "low-level" emittance. By definition, LLLT implies an output whose wavelength is within 600-980 nm and with a power less than $1,000 \mathrm{~mW}$, whereas the output used in this report had a wavelength of $808 \mathrm{~nm}$ and a power of $1,000 \mathrm{~mW}$ (White et al., 2017). This seems to suggest that highlevel laser therapy may produce even more satisfactory results in canine $\mathrm{OA}$ and potentially further improve pain management and function. 
This study has important limitations, and the lack of objective outcome measures, such as dynamic gait analysis and potentially mechanical thresholds, is the most important one. The positive effects of laser therapy were evaluated mostly based on pain scores, which are subjective and may vary depending on the observer. Moreover, the investigator who performed the VAS was aware of the treatment, and his judgment could have been biased. Unfortunately, due to the retrospective nature of this report, a randomized assignment of the dogs to different treatments, including a placebo or negative control group, as well as blinding of the clinician performing the pain assessments, was not a suitable option.

As it was evaluated by the owners and not by the clinician who performed the treatment - and considering that this scale is validated for OA-associated canine chronic pain - the CBPI might have functioned as a more reliable tool than the VAS in the dogs of this report (Brown et al., 2007). Although dog owners may have been driven by their desire to see their pet improving after therapy, owing to both the expenses incurred and psychologicalaffective implications, the unavailability of previous scores for comparison should have decreased this bias. Moreover, despite the administration of systemic analgesics was reduced in the majority of the dogs after the first laser session - a variable which may potentially have caused a subsequent worsening of the pain, the owners' satisfaction continued to increase, which further supports the hypothesis that laser therapy did produce some positive results in the study dogs.

Although the prospective study from Looney et al. (2018) could overcome the aforementioned limitations, it included a small number of subjects and was based on the subjective outcome measures. Therefore, laser therapy in dogs should still be regarded as a mostly unexplored field, and more prospective clinical trials are needed to prove the usefulness of laser therapy to treat $\mathrm{OA}$-associated pain and to refine case-specific protocols. In this perspective, the findings of the current, preliminary retrospective study contribute to provide a basis for future prospective investigations and may be used as a starting point by clinicians who aim to introduce laser therapy to their practice until more evidence is published.

\section{Author's contribution}

LB: study design, data collection and interpretation, and preparation of the manuscript; PM: intellectual contribution to manuscript preparation and critical revision; MR: contribution to data collection and revision and approval of the manuscript; CA: study design, data analysis, manuscript preparation, critical revision, and editing of the manuscript.

\section{Conflict of interest}

The authors declare that there is no conflict of interest. This research received no specific grant from any funding agency in the public, commercial, or not-forprofit sectors.

\section{References}

Brown, D.C., Boston, R.C., Coyne, J.C. and Farrar, J.T. 2007. Development and psychometric testing of an instrument designed to measure chronic pain in dogs with osteoarthritis. Am. J. Vet. Res. 68, 631-637.

Cimino Brown, D. 2017. What can we learn from osteoarthritis pain in companion animals? Clin. Exp. Rheumatol. 107, 53-58.

Huang, Z., Chen, J., Ma, J., Shen, B., Pei, F. and Kraus, V.B. 2015. Effectiveness of low-level laser therapy in patients with knee osteoarthritis: a systematic review and meta-analysis. Osteoarthr. Cartilage 23, 1437-1444.

Kennedy, K.C., Martinez, S.A., Martinez, S.E., Tucker, R.L. and Davies, N.M. 2018. Effects of low-level laser therapy on bone healing and signs of pain in dogs following tibial plateau levelling osteotomy. Am. J. Vet. Res. 79, 893-904.

Looney, A.L., Huntingford, J.L., Blaeser, L.L. and Mann, S. 2018. A randomized blind placebocontrolled trial investigating the effects of photobiomodulation therapy (PBMT) on canine elbow osteoarthritis. Can. Vet. J. 59(Suppl. 9), 959-966.

McCormack, H.M., Horne, D.J. and Sheather, S. 1988. Clinical applications of visual analogue scales: a critical review. Psychol. Med. 18, 1007-1019.

Pettitt, R.A. and German, A.J. 2015. Investigation and management of canine osteoarthritis. BMJ. 37, 1-8.

White, P.F., ElvirLazo, O.L., Galeas, L. and Cao, X. 2017. Use of electroanalgesia and laser therapies as alternatives to opioids for acute and chronic pain management. F1000Res 21(Suppl6), 2161.

Wyszyńska, J. and Bal-Bocheńska, M. 2018. Efficacy of high-intensity laser therapy in treating knee osteoarthritis: a first systematic review. Photomed. Laser. Surg. 36, 343-353.

Youssef, E.F., Muaidi, Q.I. and Shanb, A.A. 2016. Effect of laser therapy on chronic osteoarthritis of the knee in older subjects. J. Lasers Med. Sci. 7, $112-119$. 